\title{
THE E-LEARNING GRID: PEER-TO-PEER APPROACH
}

\author{
Vassil Alexandrov, ${ }^{1}$ Nia Alexandrov, ${ }^{1}$ Ismail Bhana, ${ }^{1}$ David Johnson ${ }^{1}$ \\ ${ }^{1}$ Center for Advanced Computing and Emergent Technologies, \\ University of Reading, Whiteknights, P.O. Box 225, \\ Reading, UK, RG6 6AY \\ \{v.n.,n.s,i.bhana,d.johnson \}@reading.ac.uk
}

\begin{abstract}
In this paper we present some of the approaches taken by the European E-Learning Grid consortium in building learning Grids. Some of the initial research has been done within the EC GENIUS project, which is a partnership between industry and academia. In this particular paper we focus on how in combining the collaborative and peer-to-peer approach with the relevant pedagogical paradigms we can arrive at the E-Learning Grid.
\end{abstract}

Keywords: e-Learning, peer-to-peer, Grid

\section{Introduction}

According to Equity Research (March 2000), the e-Learning market is one of the fastest growing markets worldwide. The key trends outlined are:

- Customer priorities shift away from stand-alone training, industry and customers demand a comprehensive approach to meet their needs. For example, such services as assessment and custom curriculum design, online mentoring and support reporting and tracking are in increasing demand.

- Increasing consolidation is required, since the market is very fragmented and we observe long development cycles inconsistent with the ageing of knowledge.

- Currently the global e-learning industry consists of approximately 5,000 participants offering every imaginable method of e-learning.

- Consolidation of Content, Technology and Services, in order to secure a sizable market share a complete e-learning solution has to be delivered. This can be done only in partnership. 
- Within the current E-Learning landscape there are a series of players who have developed both processes and materials to make in-roads into this potential market place. To date much of the work has been in isolation with elements of competition involved.

Against this backdrop the overarching aim of our Grid approach is "to create an ubiquitous pervasive open collaborative e-learning environment focusing on and enhancing the dialogue phases of the learning process and based on the metacognition principles. This means a learner can take a course of their choice from a common distributed virtual content repository and have it delivered to them at a time/place/format of their choice in a personalized fashion, with support available as and when they need it, from anywhere within the EU and the world respectively".

Our solution to achieve this ambitious goal is to use Grid technology to create infrastructure and collaboratory to enable different players to work together. Thus the E-Learning Grid consortium was created, which currently consists of 22 members: University of Reading, UK, University of York, UK, Trinity College, Ireland, Aristotle University of Thessaloniki, Greece, University Carlos III Madrid, Spain, INESC Porto, Portugal, INSA, Lyon, France, University of Portsmouth, UK, Emory University, USA, University of Westminster, UK, University of Salford, Johannes Kepler University, Linz, Austria, several EC Centres of Excellence and major EC research centers (Bulgarian EC Centre of Exccelence, SZTAKI, Budapest, Hungary, Cyfronet, Krakow, Poland) several major IT companies (Intel, Portugal Telecom, Support IT, Giunti Interactive Labs), Ward Education Services and ICEL Ltd, Belgium.

\section{The Approach}

We apply multifaceted approach based on the integration of novel e-pedagogy, new technologies and organizational components (see Figure 1)

Most of the partners and especially all of the partners working in the Infrastructure working group are partners of current EC funded Grid projects such as CROSSGRID and Gridlab as well as national Grid and e-Science projects.

Most of the partners are also involved in EC GENIUS project [5]. The major results from GENIUS project have shown that:

- "Delivery (which may be as technologically enhanced as we wish) is not learning nor can it imply that learning is taking place." Hence the need of new pedagogy going hand in hand with the technology and organizational components.

- Our approach is based on metacognition, e.g. the awareness of and control over one's cognitive processes. Effective thinking and learning requires 


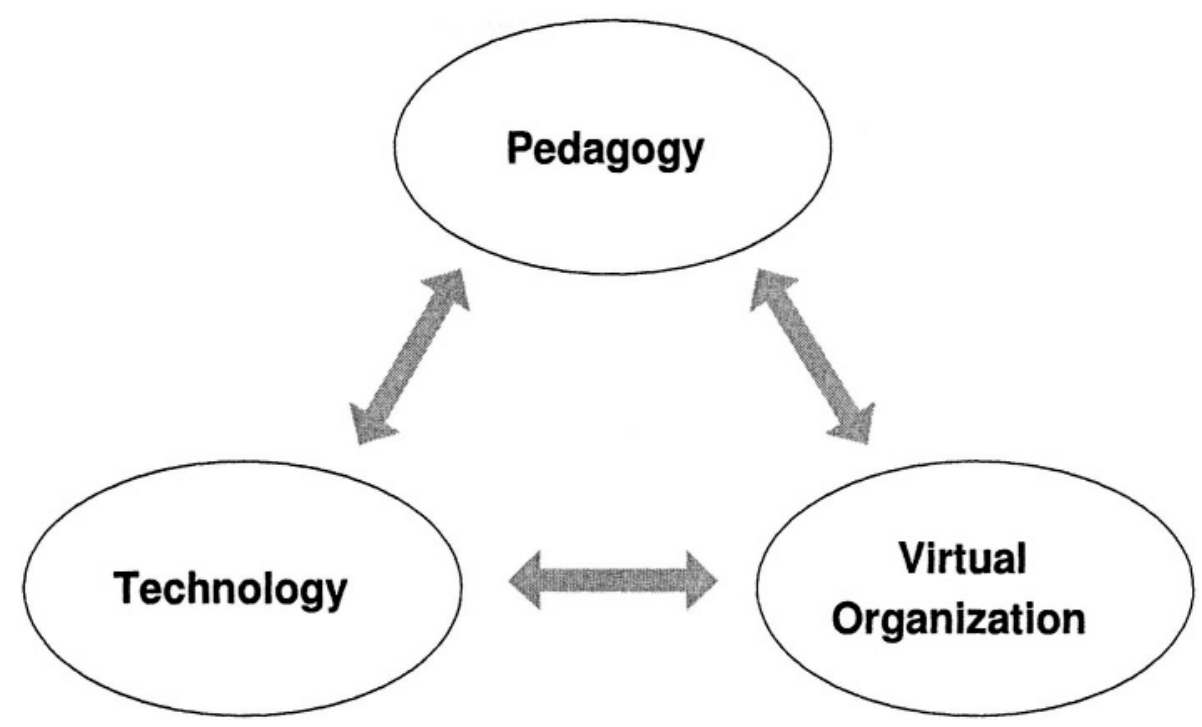

Figure 1. Our Approach.

frequent checking, goal setting, reassessing, and evaluation. We aim to improve further the quality of learning

- GENIUS also outlined the need of Organizational change and management of this change, so that we can improve quality of learning and minimize the costs.

- We should facilitate blended learning, due to the need of socialization [9] as an important element in the learning process. It should be noted that more than $70 \%$ of knowledge is not explicit, hence we need a system where socialization (exchanging knowledge in groups by social contacts) is a fundamental part of the system.

- The collaboration and collaborative environments facilitating collaborative learning and socialization are very important. Using the collaboration we can integrate workshops and independent learner groups.

- The learning should be personalized and demand driven thus being learner oriented and able to satisfy the demand for life long learning and minimize the costs through sharing of resources.

- Our experience outlined the importance of sharing of educational materials, which allows the teachers to concentrate in depth while preparing 
joint courses and at the same time minimizes the cost to the individual institutions of preparing these courses.

To translate this into practice we have to transform the conventional process of learning from constructing knowledge and acquiring skill in a traditional way to constructing knowledge using digital resources through utilizing tools and using digital communication in a virtual community (Peter Revill [4]). The management of this process and organizational clarity are also critical to this process.

In the next three sections we will give an overview of our peer-to-peer solution and finally will make some concluding remarks.

\section{Pedagogy}

Our pedagogical approach, following the achievements in [4,7], is based on the educational model which assumes that the learning process is an interactive process of seeking understanding, consisting of three fundamental components: Conceptualization, Construction and Dialogue [10]. We are focusing on Construction and Dialogue phases of the learning process, since it is known [5,10] that most of the learning is happening in these phases, and on the metacognition, which plays a significant role in developing person's learning capability. Thus we are investigating how to translate learning from theory into practice and especially in the case of e-learning, using digital tools, digital resources and digital communications $[4,5]$. Collaborative learning can facilitate the Construction and Dialogue phases of the learning process and especially: team work and a combination of individual activities, with discussion all along the learning process involving lecturers and instructors from different institutions.

On the other hand, collaborative and Grid approach allows us:

- to efficiently share resources, e.g. learning material, computing resources, lecturers and instructors.

- to efficiently reuse the learning materials based on common standard.

- to collaboratively prepare learning materials and deliver them.

All this leads to the idea of the E-Learning Grid and defining such Grid infrastructure and collaborative environment based on this infrastructure which will allow multiple Virtual Organizations (VO) to co-exist.

\section{The E-Learning GRID}

As pointed out, our recent work has identified the need for an E-Learning Grid, allowing sharing of learning materials, collaborative preparation of such materials, collaborative learning, demand driven education based on Grid ELearning services etc. It is apparent that other enabling technologies for content 
publishing, learning objects tagging to create metadata, Learning Management Systems etc are also part of the picture and essential components of the ELearning Grid.

Indeed the overview of the E-Learning Grid layered architecture is presented in Figure 2.

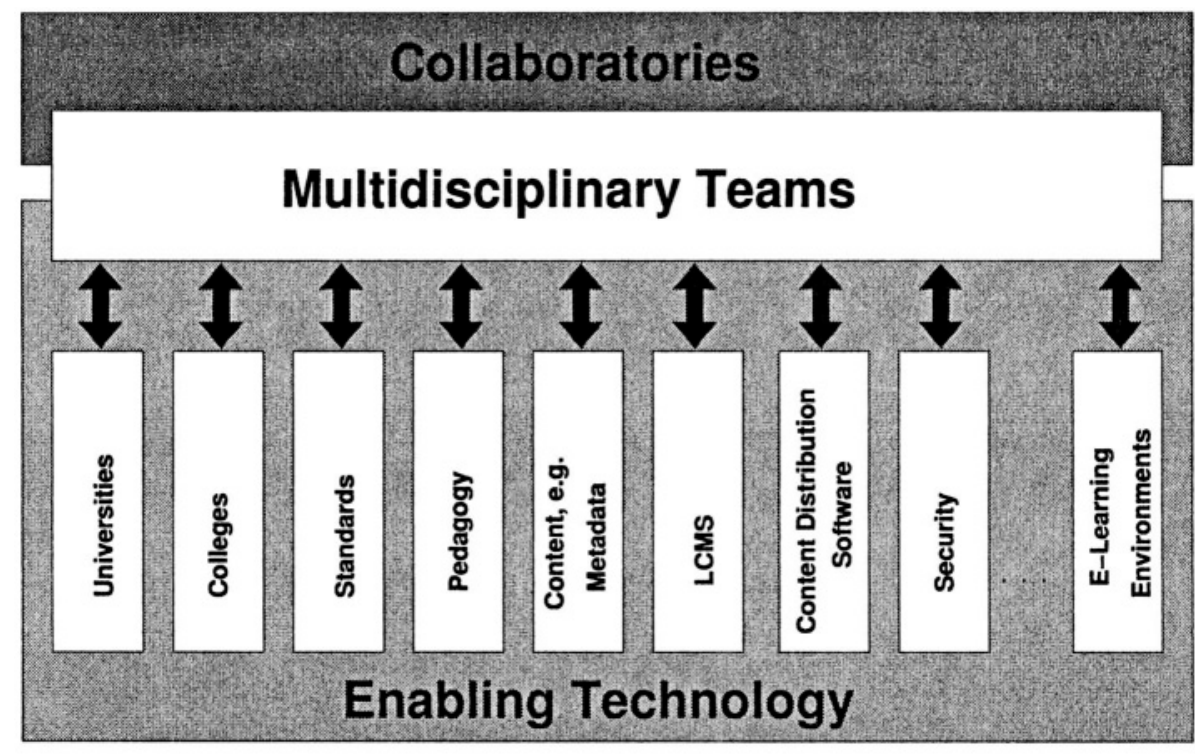

\section{GRID Infrastructure}

Figure 2. E-Learning Grid Architecture.

From our current experiments it is emerging that one approach ensuring scalability should be based on peer-to-peer technologies. Lets consider for example in more detail several layers of Figure 2.

\section{Collaboratories for the E-Learning Grid}

This involves sites in different Universities, Companies, and professional bodies, some developing content, and others developing Grid middleware, eLearning software and interfaces. We need a common framework in which to interact. Hence we need a collaborative environment in which to work and place the existing components. Our approach here is based on the Collaborative Computing Platform (Coco). Thus we will follow how the general description of the architecture presented in Figure 3 changes in this case. 
The computing landscape is shifting rapidly. Computer networks are increasingly characterised by heterogeneity and diversity as computing begins to pervade every aspect of our daily lives; new mobile technologies are juxtaposing with traditionally network technologies to become mainstream tools for communication at home and in the workplace; network connectivity is increasingly transient and dynamic. Due to the proliferation of small mobile and wireless devices and the development of a range of network protocols and middleware to support device connectivity, it is likely that in the future network heterogeneity will be the order of the day. Work groups spanning physically distant locations will increasingly rely on a whole range of tools, including personal computers, PDAs, mobile phones etc. to cooperate and coordinate their activities and to bring a sense of cohesion and proximity to teams of collaborators on a global scale.

Traditionally, applications and tools supporting collaborative computing have been designed only with personal computers in mind and support only a limited range of computing and network platforms, such as TCP/IP or UDP. These applications are not well equipped to deal with network heterogeneity and do not cope well with dynamic network topologies. The goal of this project is to develop a framework and environment that supports the creation of multi-user collaborative sessions, allowing users to self-organise and communicate, share tasks, workloads, and content, and interact across multiple different computing platforms, network platforms and operating systems when they wish to:

- Form groups with shared interests and secure domains of trust.

- Form spontaneous collaborative sessions interacting over or manipulating shared data or documents.

- Interact with others synchronously or asynchronously.

- Be aware of other users and have access to user credentials for authentication.

- Share arbitrary content using metadata representations.

We are aiming for heterogeneity in terms of both network and operating system platforms centred on two fundamental technologies: JXTA and Java.

JXTA (a project initiated by Sun Microsystems, Inc.) is a set of open network protocols that enable communication, interaction, and the sharing of resources in peer-to-peer (P2P) networks. JXTA consists of a set of network protocols that standardize the ways in which network nodes (or peers) discover other peers, resources and services, organise into groups, communicate, and monitor each other. JXTA is designed to facilitate homogenous peer connectivity within heterogeneous network environments. JXTA makes few assumptions about the network environment. JXTA specifies mechanisms not policies relating to 
the way in which peers interact in the network [6]. As a result JXTA peers are able deal with highly partitioned networks, capable of traversing firewalls and NAT, and to exist in highly volatile network environments where devices may disappear from the network with high probability. The capabilities of other peers in the network may also range greatly, from simple resource-limited devices to network servers and supercomputers. JXTA therefore provides us with interoperability at the protocol level, the kind of interoperability that is well suited to collaborative architectures designed to support a range of network platforms and operating systems.

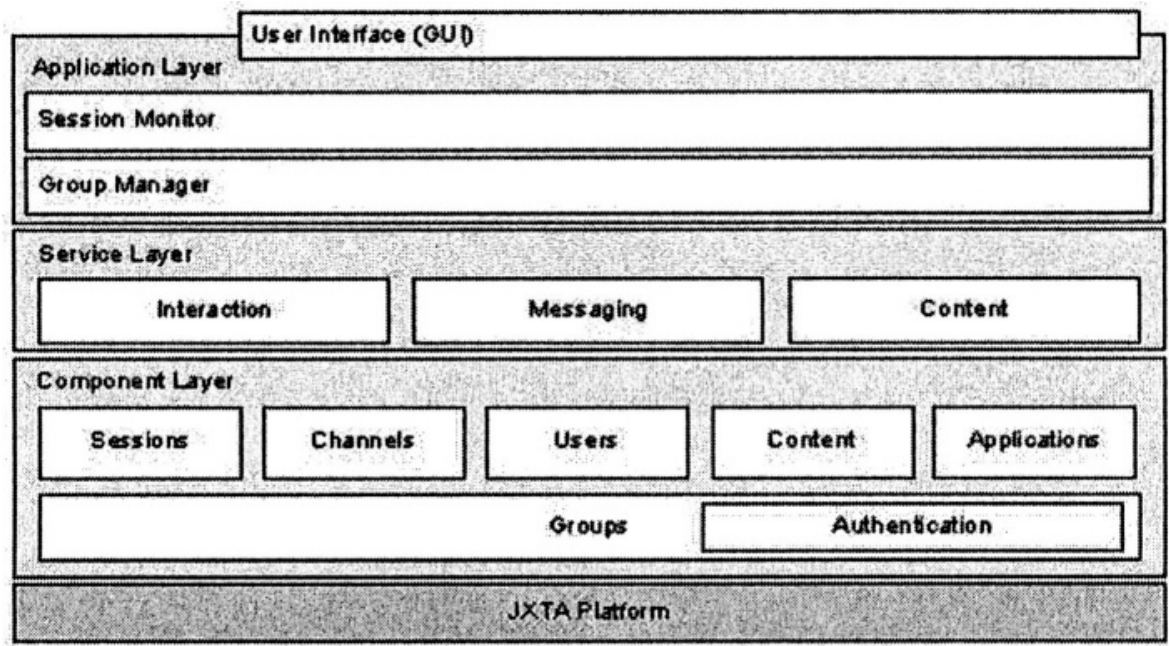

Figure 3. Coco Architecture.

Consider now the Coco architecture. Coco has been developed as both a programming environment, through a set of objects and a service APIs, and a runtime environment that allows users to create and announce collaborative sessions. The design of Coco platform is based on a layered architecture, illustrated by Figure 3.

The design focuses on three fundamental aspects that can be summarised as follows:

Components - the component layer contains a set of components that are integral to the platform. Components can be seen as manifestations of concepts and abstractions that were derived from an analysis of the system requirements. All components have an XML-based representation on the network that allows the Coco service layer to build on JXTA core services, providing the underlying propagation mechanism for the dissemination of messages between peers. 
Current components include sessions, channels, users, groups, content, and applications.

Services - services encapsulate the network service functionality of the system. The architecture is designed to provide a number of services (exposed through a set of service APIs) that enable peers to access the resources of remote peers and groups, thus forming an interface that can be built on by applications. The design consists of three core services: the interaction service, the messaging service, and the content service. The services are fundamental to the system design as they completely specify the network behaviour of peers, allowing peers to discover resources, propagate messages, and communicate in the context of a heterogeneous network environment.

Applications - the application layer provides two platform applications that allow users to create groups and to create, discover, and join collaborative sessions within the context of groups.

Layering at the architectural level encourages the encapsulation of components into modules with well-defined interfaces, enabling higher-level services to be built using the facilities of lower ones. Layering also ensures that services and applications are separated (or decoupled) and reduces dependencies within the system. The combined elements of the architecture are designed to provide an interoperable substrate that can be built on by applications via the Coco service interfaces.

Coco is currently in an early stage of development and has therefore undergone little formal or rigorous testing. However, we have been developing demonstration applications concurrently to the development of the platform, including an instant messenger with file sharing capabilities, a shared whiteboard, a shared web browser, as well as progressively developing other components.

\section{Peer-to-peer Technologies for Content Distribution}

Here we would like to give an example of an enabling technology for content distribution. Note that peer-to-peer technologies [1, 2] for content distribution e.g. pull and push, such as the embedded version of Intel's $(\AA$ Content Distribution System ( Intel $® C D S$ ). Intel ${ }^{\circledR}$ CDS can be efficiently used to distribute content. These techniques have shown to eliminate the bandwidth bottleneck for cutting edge multimedia and e-Learning. Today e-Learning content developers are constrained in their design as they need to design for the narrowest pipe which transports an e-Learning module to a user. The use of client caching and peer-to-peer protocols dramatically improves file download times while reducing infrastructure costs [2]. This technology has been tested in an intranet environment with great results and in a wide area networks, where through GENIUS we have focused on the internet use of this technology and 
we expect it's broad deployment to enable a high performing cost effective e-Learning Grid.

\section{Building Virtual Organizations}

In the previous sections we have considered the pedagogical and technological components, Here we will very briefly focus on the organizational aspects. The above infrastructure allows us high degree of flexibility:

- It offers scalable solution, partners can join and various components such as modules etc being placed in the environment.

- Offers possibility to form groups with shared interests.

- Offers possibility to form collaborative sessions interacting over or manipulating shared data or documents.

- Interact with others synchronously or asynchronously.

Based on the above we can form different Virtual Organizations which can co-exist for delivering specific modules, courses etc. The collaborative capabilities allow facilitation of collaborative learning, collaborative preparation of materials etc.

\section{Conclusion}

In this paper we have presented a peer-to-peer approach to the E-Learning Grid. We have outlined a layered collaborative computing platform facilitating most of the functionality required for collaborative learning on the Grid.

\section{Acknowledgments}

The authors wish to thank Martin Curley, Intel Ireland Ltd, Leixlip, Co. Kildare Republic of Ireland, for his contribution to the paper with the material on Intel's ${ }^{\circledR}$ peer-to-peer content distribution system (Intel ${ }^{\circledR} \mathrm{CDS}$ ). The authors also wish to thank, DG Education and Culture of the EC for their partial support for the work through the EC GENIUS project: 2001-3450/001-001 EDU-ELEARN.

\section{References}

[1] A. Oram, Peer-to-Peer: Harnessing the Power of Disruptive Technologies, O'Reilly, March, 2001.

[2] Martin O. Curley, "Peer-to-Peer Computing Enabled Collaboration", Proc. of ICCS2002, LNCS 2330, Springer-Verlag, 646-654.

[3] Nia Alexandrov, J.S.Pascoe, and, Vassil Alexandrov "Collaborative Computing and ELearning”. Proc of ICCS2002, LNCS2330, Springer-Verlag, 688-694. 
[4] Peter Revill, "GENIUS project Pedagogical Report", 2003, www.genius.reading.ac.uk

[5] GENIUS - Generic E-Learning Environments and Paradigms for the New Pan European Information and Communication Technologies Curricula, EC project, 2001 - 2003, www.genius.reading.ac.uk

[6] A.B. Gong, L (2002): Project JXTA: A Technology Overview, Palo Alto, CA, USA: Sun Microsystems, Inc.,2002.http://www.jxta.org/project/www/docs/jxtaview_01nov02.pdf.

[7] Ian Foster, Carl Kesselman, Steven Tuecke, "The Anatomy of the Grid", http://www.globus.org/research/papers/anatomy.pdf

[8] Ian Foster, Carl Kesselman, Jeffrey M. Nick, Steven Tuecke, "The Physiology of the Grid", http://www.globus.org/research/papers/ogsa.pdf

[9] Nonaka, The Knowledge Creating Company, Harvard Business Review No. 91608, 1991

[10] Mayes, T., "Pedagogy, Lifelong Learning and ICT", A discussion paper for IBM Chair presentation, Glasgow 18 May, 2000. 